$\underline{\text { Preprint typeset in JHEP style - HYPER VERSION }}$

hep-th/0205106

DAMTP-02-51

$\mathrm{IC} / 2002 / 27$

\title{
Supergravity solutions for D-branes in Hpp-wave backgrounds
}

\author{
P. Bain \\ DAMTP, University of Cambridge, \\ Centre for Mathematical Sciences, Wilberforce Road, \\ Cambridge CB3 0WA, UK \\ E-mail: P.A.Bain@damtp.cam.ac.uk

\section{P. Meessen} \\ International School for Advanced Studies, \\ Via Beirut 2-4, \\ 34014 Trieste, ITALY \\ E-mail: meessen@sissa.it
}

\author{
M. Zamaklar \\ The Abdus Salam ICTP, \\ Strada Costiera 11, \\ 34014 Trieste, ITALY \\ E-mail: mzama@ictp.trieste.it
}

\begin{abstract}
We derive two families of supergravity solutions describing D-branes in the maximally supersymmetric Hpp-wave background. The first family of solutions corresponds to quarter-BPS D-branes. These solutions are delocalised along certain directions transverse to the pp-wave. The second family corresponds to the nonsupersymmetric D-branes. These solutions are fully localised. A peculiar feature of the nonsupersymmetric solutions is that gravity becomes repulsive close to the core of the D-brane. Both families preserve the amount of supersymmetry predicted by the D-brane probe/CFT analysis. All solutions are written in Brinkman coordinates. To construct these kind of solutions it is crucial to identify the coordinates in which the ansatz looks the simplest. We argue that the natural coordinates to get the supergravity description of the half-BPS branes are the Rosen coordinates.
\end{abstract}




\section{Contents}

1. Introduction and Summary 1

2. Setting up an ansatz 4

2.1 The geometry of the Hpp-wave and D-brane embeddings 1

2.2 The ansatz for quarter-BPS D-branes 8

3. Supersymmetry analysis 11

4. Solving the field equations 14

4.1 Supersymmetric solutions

4.2 Non-BPS solutions 18

5. Discussion 19

A. Some technical details 22

B. Probe analysis 24

\section{Introduction and Summary}

Recent developments of the AdS/CFT correspondence [1] have involved the study of a particular class of supergravity pp-waves [2, 3]. These geometries naturally arise by taking the so-called Penrose limit of various near horizon regions of D-branes. The Penrose limit [4, 2] is a general procedure in which, by a suitable rescaling of coordinates and parameters characterising a (super)gravity solution, one focuses on the region close to an arbitrary null geodesic. The scaling is performed in such a way that the zoomed part of the space still solves the equations of motion. In [4] Penrose proved that this procedure leads, for any gravity background, to a gravitational pp-wave. The extension of his arguments to supergravity solutions, which include additional higher rank forms, leads to generalisations of gravitational ppwaves, which are, in addition to a nontrivial metric, characterised by fluxes of NSNS or/and RR fields.

Waves which arise as Penrose limits of supergravity solutions for various branes are also interesting from the pure supergravity point-of-view since they usually preserve a fraction of supersymmetry larger than one half [5, 6]. In particular, the 
Hpp-wave which will be considered in this paper is a maximally supersymmetric vacuum of the type IIB string theory.

The main obstacle in proving the conjectured AdS/CFT duality [7, 8, 9 $]^{1}$ lies in the difficulty to quantise strings in AdS spaces. The beauty of the proposal of [1], 11] is that it suggests a relatively simple class of supergravity backgrounds where one can explicitly quantise strings [12, 13] and "identify" a subsector of the corresponding gauge theory duals. In [14] it was shown that taking the Penrose limit of the neighborhood of the null geodesics along the 5-sphere in $A d S_{5} \times S^{5}$ space leads to a maximally supersymmetric gravitational pp-wave with a non vanishing RR flux (2.1) [3]. Furthermore, it was argued that string excitations in this background correspond to the subsector of the Yang-Mills theory characterised by large $\mathrm{R}$-charges. These states are dual to the string excitations in the initial $A d S$ space that carry large angular momentum $(J \leftrightarrow R)$ in one of the directions of the $S^{5}$.

In the AdS/CFT correspondence, D-branes usually correspond to nonperturbative objects on the gauge side or defects on which a lower dimensional conformal field theory lives. In order to study the field theory dynamics in the presence of these objects using the duality, it is useful to have access to the supergravity solutions for D-branes in $A d S$ spaces. Unfortunately, not much is known explicitly about these solutions. There are two methods that one might try to construct these solutions : by directly writing the appropriate ansatz for D-branes in the $A d S$ space, or by taking an appropriate near horizon limit of the supergravity solutions for intersecting D-branes. The problem with the second approach is that fully localised supergravity solutions for D-brane intersections are usually not known. The only exceptions are [15]-[19]. Although the simplified ansatzes that are often used in the literature preserve the right amount of supersymmetry, they necessarily require that at best one of the intersecting D-branes is smeared along the worldvolume directions of the other D-brane ${ }^{2}$. These are the so-called partially localised solutions. However, even in these cases, the solutions take a very complicated form [17]. Some simplification occurs if one replaces the harmonic function for a smeared brane by its near horizon expression [20]. However, these solutions then describe D-branes in the near horizon geometry of the smeared D-branes.

Taking the Penrose limit simplifies some of the problems that are present for $A d S$ spaces. For instance, while the CFT construction of D-branes in $A d S$ spaces is rather complicated [21, 22, 23] it is much easier to treat this problem in pp-wave backgrounds [24, 25]. So one might hope that constructing D-brane supergravity solutions in these backgrounds is also more tractable. Performing this exercise would be useful, since it could teach us how to attack the similar problem in $A d S$ spaces.

\footnotetext{
${ }^{1}$ For a more complete list of references in this area see [10].

${ }^{2}$ The only cases where this is not true is when there are no relative transverse directions for one of the D-branes, namely for a D-brane sitting inside an higher dimensional D-brane or when there are no overall transverse directions.
} 
As in $A d S$ spaces, there are two strategies that one can adopt to find the solution. In one approach, one starts with the solution for a D-brane in an $A d S$ space and takes its Penrose limit. This technique has been used recently in [26, 27], starting from the supergravity solution 19] for $D 5 / M 5$ branes wrapping the $A d S_{3} \times S^{3}$ space. The resulting configurations correspond to localised solutions for $D 5 / M 5$-branes in ppwave backgrounds. ${ }^{3}$. Related work on the D/M-branes in these pp-wave backgrounds has been done in [28].

The pp-wave which will be considered in this paper is characterised by a non vanishing 5-form RR flux and arises as the Penrose limit of the $A d S_{5} \times S^{5}$ space. Since at present there are no known solutions describing D-branes in this space, one cannot use the previous approach. Therefore, the method we will adopt is to directly write an ansatz for the D-brane in the pp-wave background.

Recently, in [29], various embeddings of D-branes in the Hpp-wave geometry were explored using the D-brane probe approach. There are three different families of D-branes in Hpp-waves : longitudinal D-branes for which the pp-wave propagates along the worldvolume of the D-brane, transversal D-branes for which the pp-wave propagates in a direction transverse to the D-brane but the timelike direction is along its worldvolume and instantonic D-branes for which both the direction in which the pp-wave propagates and the timelike direction are transverse to the D-brane. In this paper we consider supergravity solutions for longitudinal D-branes only.

In order to write an ansatz for the D-branes in the Hpp-wave background, we first study in section 2.1 the geometry of this space and various brane embeddings. One important property is that, due to the nonisotropy of the space, D-branes with different orientations can preserve different amounts of supersymmetry. Another point is that the pp-wave is homogeneous space, hence all points in the space are equivalent. However, this property is not manifest in most coordinate systems, and naively it looks like the coordinate origin is a physically distinct point. The homogeneity in particular implies that all types of branes can pass through any point in space at a given moment in time. It can happen, however, that their embedding when passing through the origin for example, looks much simpler than when passing through an arbitrary point in space. We demonstrate this explicitly on the example of $1 / 2$ and 1/4-BPS D-branes.

The first step of writing the ansatz, consists in identifying "natural" coordinates in which the ansatz looks the simplest. By rewriting the embedding of different probe D-branes in various coordinate systems, we first identify in section 2.1 the Brinkman coordinates as the natural coordinates for describing 1/4-BPS and nonsupersymmetric D-branes. In section 2.2 we write an ansatz in these coordinates. We also argue that the natural coordinates for the $1 / 2$-BPS D-branes are the Rosen coordinates.

\footnotetext{
${ }^{3}$ Note that it does not make much sense to take the Penrose limit of the delocalised solutions of [20], since they do not correspond to (delocalised) D-branes deforming an $A d S$ space.
} 
One of the main characteristics and perhaps limitations of our ansatz is that the metric is diagonal in Brinkman coordinates. This property will force us to delocalise the supersymmetric solutions along some directions transverse to the brane when solving the equations of motion. However, these restrictions have to be imposed only on the harmonic function characterising the D-brane, and not on the function characterising the pp-wave. Hence, all our solutions asymptotically tend to the unmodified Hpp-wave. Also, despite the simplicity of the ansatz, the non-supersymmetric solutions that we find are fully localised.

In section 3 we analyse the supersymmetry preserved by our ansatz according to the orientation of the D-brane and we find agreement with the CFT/probe brane analysis. In section 4 we solve the supergravity equations and give the explicit form of the solutions, including the non-supersymmetric ones. Those exhibit a repulsive behavior close to the D-brane, even for the non-singular D3-brane solution. The important issue of stability of these branes is currently under investigation using the probe brane/CFT approaches. We conclude with comments on open questions. Finally, we attach two appendices. In the first one, we present some technical details of the calculations. In the second appendix, we repeat some of the D-brane probe analysis of [29] with special emphasis on the nonsupersymmetric branes, in order to make a clear parallel between the supersymmetry analysis from the probe brane and supergravity points-of-view.

\section{Setting up an ansatz}

\subsection{The geometry of the Hpp-wave and D-brane embeddings}

To set up an ansatz suitable to obtain the supergravity solution describing a D-brane in the Hpp-wave background of [3], it is important to first understand the geometry of this space. The metric part of the solution has isometry group $S O(1,1) \times S O(8)$ which is further broken down to $S O(1,1) \times S O(4) \times S O(4)$ by the presence of a null $\mathrm{RR} 5$-form flux. As mentioned in the introduction, this space is nonisotropic and homogeneous.

The main difficulty in constructing these supergravity solutions consists in identifying a coordinate system where the description of the D-brane is the simplest. This is similar to the problem that one would face if one would only know the Minkowski space in spherical coordinates and try to describe flat D-branes in these coordinates. Cartesian coordinates are the natural coordinates to describe infinite D-branes in flat space. So the question that one should first ask is what are the analogues of the Cartesian coordinates for D-branes in pp-wave backgrounds? The answer to this question is more complicated than in flat space, and as we will now see it depends very much on what kind of D-branes one considers. 
Some possible supersymmetric embeddings of D-branes in the Hpp-wave spacetime have been explored in [29, 30] using the D-brane probe and the CFT approaches respectively. Both analyses were performed in Brinkman coordinates, in which the pp-wave metric and 5-form flux read

$$
\begin{aligned}
& d s^{2}=2 d u(d v+S d u)-d \vec{z}_{(8)}^{2}, S=-\frac{W^{2}}{32} z^{\mu} z_{\mu}, \\
& F_{(5)}=W d u \wedge\left(d z^{1} \wedge d z^{2} \wedge d z^{3} \wedge d z^{4}+d z^{5} \wedge d z^{6} \wedge d z^{7} \wedge d z^{8}\right),
\end{aligned}
$$

where the directions transverse to the pp-wave are denoted by $z^{\mu}=z^{1}, \ldots, z^{8}$. If we embed a $\mathrm{D} p$-brane in this background in such a way that the pp-wave propagates along its worldsheet, the worldvolume coordinates split into three sets : the "lightcone coordinates" 4 and $v, m$ coordinates along the first $S O(4)$ subspace $z^{1}, \ldots, z^{4}$ and $n$ coordinates along the second $S O(4)$ space $z^{5}, \ldots, z^{8}$. For a $\mathrm{D} p$-brane $(m+n=p-1)$ with such orientation we adopt the notation of [29] and denote such embeddings with $(+,-, m, n)$.

One of the conclusions of [29] is that there are three families of longitudinal, infinite, flat (in Brinkman coordinates) D-branes with no worldvolume fluxes that sit at the origin of the pp-wave. The number of supersymmetries that they preserve depends on their orientation with respect to the isometry group $S O(4) \times S O(4)$ :

- 1/2-BPS D-branes with the embedding $(+,-, m+2, m)$, for $m=1, \ldots, 4$,

- 1/4-BPS D-string with the embedding $(+,-, 0,0)$,

- non-supersymmetric D-branes with the embedding $(+,-, m, m)$ for $m=1,2,3$.

In [29], it was also noticed that to rigidly move the first type of D-brane away from the origin and to preserve the same amount of supersymmetries, one has to turn on a worldvolume flux whose value is equal to the distance of the D-brane from the origin. By rigidly we mean here that the D-brane is located at a constant transverse position $y^{\hat{a}}$. For any other value of the flux, including zero, a D-brane that is rigidly sitting away from the origin in Brinkman coordinates is only 1/4-BPS.

However, it is important to realise that since the space is homogeneous, there is nothing physically special about the origin of the space. This means that all listed 1/2-BPS and 1/4-BPS branes can pass through any point in the transverse space at a given moment in time. At each such point there is an infinite number of $1 / 2$ BPS and an infinite number of 1/4-BPS D-branes, labelled by different worldvolume fluxes. However, the embedding of many of these D-branes looks quite complicated when they are not positioned at the coordinate origin. We will see this explicitly on the example of the half and quarter-BPS D-branes with no worldvolume fluxes. In the remaining of the paper we only consider D-branes with no worldvolume fluxes.

\footnotetext{
${ }^{4}$ Strictly speaking $(u, v)$ coordinates are not lightcone; only the direction $v$ is null, while $u$ is timelike for $S \neq 0$.
} 
From the D-brane probe analysis we have learnt that 1/4-BPS D-branes have a simple description in Brinkman coordinates, independent of their position with respect to the origin. This is an important property, since in order to find supergravity solutions for the simple ansatz that we use, we are forced to smear several of these solutions in some directions. This is the same type of restriction that one faces when constructing supergravity solutions for intersecting D-branes, with a simple diagonal ansatz [20, 34]. The smearing procedure physically means that one is constructing an array of D-branes of the same type with an infinitesimally small spacing. However, the probe brane results tell us that, unless we turn on additional bulk fluxes (sourced by the worldvolume fluxes of the $1 / 2$-BPS D-branes), a periodic array of rigid D-branes in Brinkman coordinates with orientation $(+,-, n+2, n)$ will be only one quarter supersymmetric! In conclusion, Brinkman coordinates are coordinates in which one expects the (smeared) supergravity solution for the 1/4-BPS D-branes to look the simplest.

What about 1/2-BPS D-branes? As we will see the 1/2-BPS D-brane located away from the origin of the Brinkman coordinates has a complicated shape (see equation (2.4)). Hence, to describe an array of these D-branes in Brinkman coordinates, it is not clear what ansatz one should use. In Rosen coordinates on the other hand, where the homogeneity property of the space transverse to the pp-wave is manifest, these 1/2-BPS D-branes look very simple, independent of the position with respect to the origin. In Rosen coordinates, the metric and the 5-form of the pp-wave take the form

$$
\begin{aligned}
& d s^{2}=2 d \tilde{u} d \tilde{v}-\cos ^{2}(\alpha \tilde{u})(d \overrightarrow{\tilde{z}})^{2} \\
& F_{[5]}=W \cos ^{4}(\alpha \tilde{u}) d \tilde{u} \wedge\left(d \tilde{z}^{1} \wedge d \tilde{z}^{2} \wedge d \tilde{z}^{3} \wedge d \tilde{z}^{4}+d \tilde{z}^{5} \wedge d \tilde{z}^{6} \wedge d \tilde{z}^{7} \wedge d \tilde{z}^{8}\right),
\end{aligned}
$$

where we have defined $\alpha=W / 4$. These are related to the Brinkman coordinates (2.1) by the change of variables

$$
u=\tilde{u}, \quad v=\tilde{v}-\frac{\alpha}{4} \tilde{z}^{2} \sin (2 \alpha \tilde{u}), \quad z=\tilde{z} \cos (\alpha \tilde{u}) .
$$

If we denote with $y^{\hat{a}}$ the directions transverse to the D-brane, we see that the $1 / 2$-BPS D-brane sitting at a position $y^{\hat{a}}=0$ in the coordinate system (2.1) has an embedding $\tilde{y}^{\hat{a}}=0$ after the change of coordinates. However, we can now shift the origin of the coordinate system by an arbitrary vector $\vec{z}_{0}: \tilde{z}^{\mu} \rightarrow \tilde{z}^{\mu}+z_{0}^{\mu}$ for $\mu=1, \ldots, 8$, and this $1 / 2$-BPS D-brane will sit at a position $\tilde{y}^{\hat{a}}=y_{0}^{\hat{a}}$. Hence, we see that in Rosen coordinates, 1/2-BPS D-branes always have a "flat" embedding, independently of the choice of an origin.

To determine the shape of this D-brane in the coordinates (2.1), we have to perform a further change of coordinates (2.3) with angle $\tilde{u}$. This gives us an embedding of the 1/2-BPS D-branes in coordinates (2.1) away from the origin of the space; 
the $p+1$-dimensional surface that they describe is defined by the following $9-p$ constraints

$$
y^{\hat{a}}=y_{0}^{\hat{a}} \cos (\alpha \tilde{u}) .
$$

So, although 1/2-BPS D-branes that pass through the origin in Brinkman coordinates look "flat", once we move them away from the origin, their shape is much more complicated $^{5}$. The shapes of $1 / 2$ and $1 / 4$-BPS D-branes in Brinkman and Rosen coordinates are depicted in figure 1 .

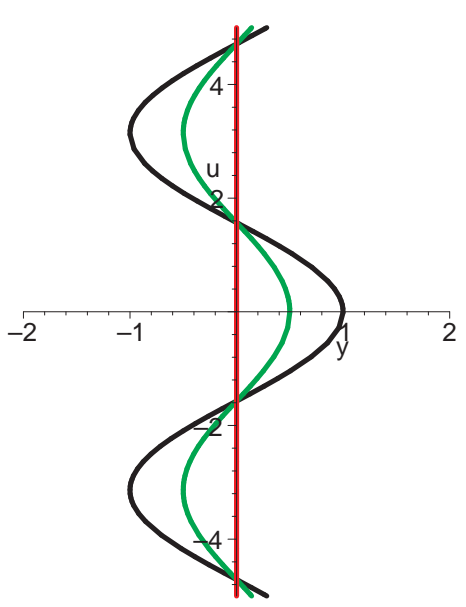

1/2-BPS branes in Brinkman coordinates

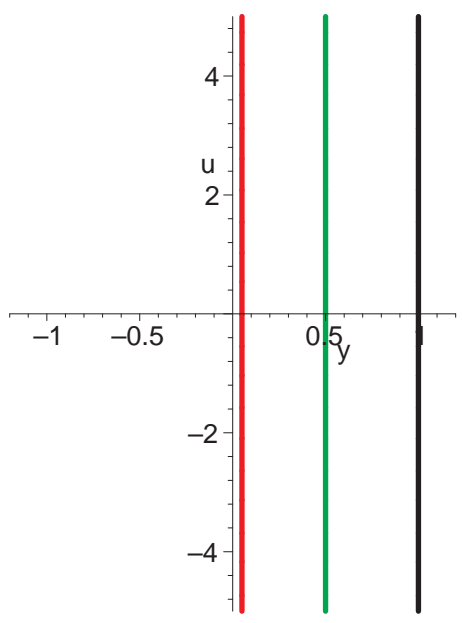

1/4-BPS branes in Brinkman coordinates

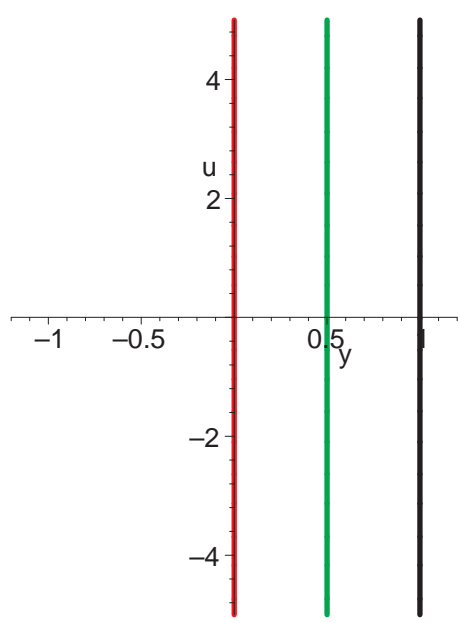

1/2-BPS branes in Rosen coordinates

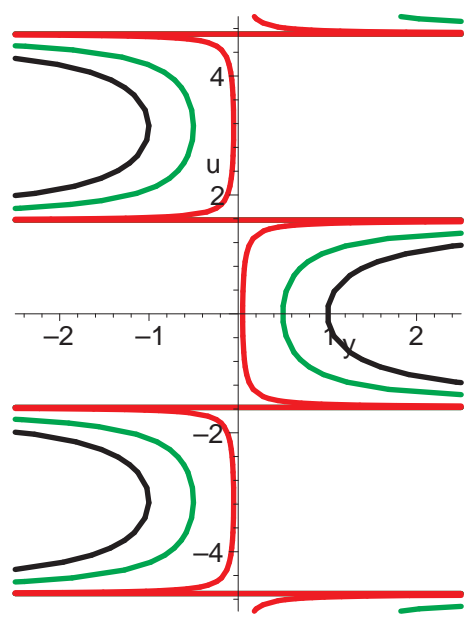

1/4-BPS branes in Rosen coordinates

Figure 1: The shapes of $1 / 2$ and 1/4-BPS D-branes in Brinkman and Rosen coordinates. The vertical axes correspond to the time-like coordinate $u$, while the horizontal axes correspond to the directions transverse to the brane.

\footnotetext{
${ }^{5}$ The procedure that we have just described is completely analogous to what one has to do in Minkowski space to change the origin of a cylindrical system of coordinates. One first passes to Cartesian coordinates, shifts the origin of the space and then goes back to cylindrical coordinates.
} 
This preliminary discussion leads us to expect that the natural coordinates one should use to write the (smeared) supergravity ansatz for the 1/2-BPS D-branes are Rosen coordinates. However, a quick inspection reveals that the "naive", general diagonal ansatz that one can write in these coordinates does not seem to allow for the $1 / 2$-BPS solutions. We are currently investigating more general ansatzes in these coordinates.

Finally, it is interesting to note that the worldsheets of $1 / 2$-BPS D-branes are geodesic submanifolds (the extrinsic curvature of the worldsheet vanishes). This is similar to situation in Minkowski space where maximally supersymmetric D-branes are flat sheets spanned by straight lines, i.e. Minkowski geodesics. The main difference is that geodesics in this space are not straight lines in Brinkman coordinates. They are curves, depicted in figure 1 1a and given by equations

$$
z=A \cos (\alpha u), \quad v=B u+C \sin (2 \alpha u),
$$

where $A, B$ and $C$ are constants determined in terms of lightcone momenta and energy. Comparing this expression with (2.3) we see that going from Brinkman to Rosen coordinates means that we are passing to a coordinate system with the coordinate grid given by the geodesics of a point particle (up to a rotation in the $u-v$ plane). It is now clear why a $1 / 2$-BPS D-brane in Rosen coordinates has a flat embedding, independent of the point through which it passes.

Note also that the fact that all geodesics starting at the origin intersect at $u=\frac{\pi}{2 \alpha}$ implies that there are apparent coordinate singularities at $u=\frac{\pi}{2 \alpha}+\frac{n \pi}{\alpha},(n \in \mathbb{Z})$ in Rosen coordinates.

Finally, before writing the ansatz, to set up our conventions, we give the action and the supersymmetry variations that we will need. The relevant part of the type IIB action is

$S=\int d x^{10} \sqrt{g}\left\{e^{-2 \phi}\left(R-4(\partial \phi)^{2}\right)+\frac{1}{2}\left(\partial C_{[0]}\right)^{2}+\frac{1}{4 \cdot 3 !}\left(F_{[3]}\right)^{2}+\frac{1}{4 \cdot 5 !}\left(F_{[5]}\right)^{2}+\frac{1}{4 \cdot 7 !}\left(F_{[7]}\right)^{2}\right\}$.

where the 5 -form field strength is self-dual and satisfies the Bianchi identity $d F_{(5)}=0$. The corresponding supersymmetry variations are

$$
\begin{aligned}
\delta \Psi_{\mu} & =D_{\mu} \epsilon+\frac{1}{16} e^{\phi}\left(2 \not \partial C_{[0]}\left(i \sigma^{2}\right)+\frac{1}{3 !} \mathscr{F}_{[3]}\left(\sigma^{1}\right)+\frac{1}{5 !} \mathbb{F}_{[5]}\left(i \sigma^{2}\right)+\frac{1}{7 !} \mathscr{F}_{[7]}\left(\sigma^{1}\right)\right) \gamma_{\mu} \epsilon \\
\delta \chi & =\not \partial \phi \epsilon+\frac{1}{4} e^{\phi}\left(-4 \not \partial C_{[0]}\left(i \sigma^{2}\right)-\frac{1}{3 !} \not F_{[3]}\left(\sigma^{1}\right)+\frac{1}{7 !} \not \mathbb{F}_{[7]}\left(\sigma^{1}\right)\right) \epsilon,
\end{aligned}
$$

where we have used the doubled formulation of supergravity with both electric and magnetic RR fields, except for the 0 -form $C_{[0]}$. Therefore, for the $D 7$-branes, we will express our results using $C_{[0]}$.

\subsection{The ansatz for quarter-BPS D-branes}

Motivated by the analysis in the previous section, we use Brinkman coordinates to write an ansatz for 1/4-BPS and nonsupersymmetric D-branes. For the metric part 
of the ansatz, we write a simple standard metric for a superposition of D-branes with pp-waves. For a pp-wave propagating in the directions $u$ and $v$ along the worldvolume of the D-brane this ansatz reads

$d s^{2}=H\left(y, y^{\prime}\right)^{-\frac{1}{2}}\left(2 d u\left(d v+S\left(x, x^{\prime}, y, y^{\prime}\right) d u\right)-d \vec{x}^{2}-d \vec{x}^{2}\right)-H\left(y, y^{\prime}\right)^{\frac{1}{2}}\left(d \vec{y}^{2}+d \vec{y}^{2}\right)$.

The metric is given in the string frame, and the D-brane worldvolume coordinates are $\left(u, v, x^{i}=x^{1}, \ldots, x^{m}, x^{I}=x^{1}, \ldots, x^{\prime n}\right)$, while directions transverse to the D-brane are $\left(y^{a}=y^{1}, \ldots, y^{(4-m)}, y^{\prime A}=y^{\prime 1}, \ldots, y^{(4-n)}\right)$. Note that the function $H$ characterising the D-brane is at this stage allowed to depend on all transverse coordinates $y, y^{\prime}$.

The components of the Ricci tensor for this metric are given in appendix $\mathrm{A}$. An important observation is that all components of the Ricci tensor, save $R_{u u}$, are independent of the function $S$ appearing in the metric. The easiest way to ensure that the equations of motion are satisfied is then to keep the RR field and the dilaton coming from the D-brane unchanged. The components of the 5-form flux of the ppwave which are kept nonzero are the same as in the absence of the D-brane. Hence, we make the following ansatz for the RR field strength and the dilaton

$$
\begin{aligned}
F_{[p+1]} & =d u \wedge d v \wedge d x^{1} \wedge \cdots \wedge d x^{m} \wedge d x^{11} \cdots \wedge d x^{\prime n} \wedge d H^{-1} \\
F_{[5]}^{\mathrm{w}} & =F_{[5]}^{(1)}+* F_{[5]}^{(1)} \\
F_{[5]}^{(1)} & =W\left(z^{\mu}\right) d u \wedge\left(d x^{1} \wedge \cdots \wedge d x^{m} \wedge d y^{1} \wedge \cdots \wedge d y^{(4-m)}\right), \\
e^{\phi} & =H^{\frac{3-p}{4}}
\end{aligned}
$$

where ' $*$ ' in $F_{[5]}$ denotes the Hodge duality with respect to the metric (2.8) and $W(z)$ is an undetermined function which can depend on all directions transverse to the pp-wave. Also, in the case of the D3 brane, one has to add to the form (2.9) its Hodge dual. Next, we use the fact that $F_{[5]}$ has to satisfy the Bianchi identity. This condition, supplemented with the self-duality of $F_{[5]}$, is enough to ensure that the equation of motion for $F_{[5]}$ is satisfied. The requirement that $F_{[5]}$ is closed implies the following set of equations

$$
\begin{aligned}
\partial_{x^{\prime}} W & =0, & & \partial_{y^{\prime}} W=0, \\
\partial_{x}\left(W H^{\frac{(m-n)}{2}}\right) & =0, & & \partial_{y}\left(W H^{\frac{(m-n)}{2}}\right)=0 .
\end{aligned}
$$

The first two conditions lead to the restriction $W=W(x, y)$, while the third one, when supplemented with $H=H\left(y, y^{\prime}\right)$, shows that $W=W(y)$. Finally, the last condition implies

$$
\begin{array}{ll}
H=\left(W^{-1}(y) \hat{H}\left(y^{\prime}\right)\right)^{\frac{2}{(m-n)}}, & (m \neq n) \\
H=H\left(y, y^{\prime}\right), \quad W=\text { const. }, & (m=n),
\end{array}
$$

where $\hat{H}$ is an arbitrary function of $y^{\prime}$. When $m \neq n$, the D-brane "harmonic" function has a multiplicative dependence on the transverse coordinates. Usually, 
this kind of functional dependence cannot be matched into a delta function brane source [35], and hence one is then forced to drop dependence of $H$ on $y$ or $y^{\prime}$ and consider only a delocalised solution. We will show now that consistency of our ansatz with the equations of motion also implies such smearing.

Let us now consider the constraints coming from the remaining supergravity equations. Inspection of the dilaton equation of motion (A.1) shows that the dilaton does not couple to the pp-wave function $S$ and that it is not modified by its 5 -form flux. This equation implies that $H$ is a harmonic function in the space transverse to the D-brane.

The equations of motion for the RR fields and the Bianchi identities can be all written in a nice compact form given in the appendix, see (A.5). Since we are looking for supergravity solutions with vanishing NSNS B-field, we have to ensure that the Chern-Simons (CS) term in the Bianchi identity for $H_{[7]}$ vanishes

$$
d H_{[7]}=-\frac{1}{2}{ }^{\star} F \wedge F .
$$

It easy to check that, with our ansatz, the right hand side is zero for the $D 1, D 3$ and $D 7$-branes. For the $D 5$-brane, the following embeddings are possible with the corresponding CS terms (coming from $* F_{[7]} \wedge F_{[5]}$ term),

$$
\begin{aligned}
& (+,-, 4,0): C S \sim \epsilon_{A B C D} \partial_{D}\left(H^{-1}\right) d x^{A} \wedge d x^{B} \wedge d x^{C} \wedge d u \wedge d x^{1} \wedge d x^{2} \wedge d x^{3} \wedge d x^{4} \\
& (+,-, 3,1): C S \sim\left(\partial_{y} H\right) d y^{\prime 1} \wedge d y^{\prime 2} \wedge d y^{\prime 3} \wedge d u \wedge d x^{1} \wedge d x^{2} \wedge d x^{3} \wedge d y \\
& (+,-, 2,2): C S=0 .
\end{aligned}
$$

In the first case, we see that unless the function $H$ is trivial, our ansatz without NSNS fields is incompatible with the equations of motion and Bianchi identities. Using the probe brane approach, one can also check that a D5-brane with a $(+,-, 4,0)$ embedding is consistent only if one allows for non-trivial worldvolume fluxes without or with non-constant transverse scalars fields. This last possibility arises as the Penrose limit of a D5-brane corresponding to the baryon vertex in the gauge theory dual [36]. In this case, one expects to have spikes due to fundamental strings stretched between the D5-branes wrapped on $S^{5}$ and the D3-branes whose near horizon geometry generates $A d S_{5} \times S^{5}$. The supergravity manifestation of this phenomenon is the presence of non-trivial NSNS fluxes induced by the contribution of the RR fields to the CS couplings which presumably survives after one takes the Penrose limit. However, we will not discuss this kind of solutions.

The second embedding is consistent with our ansatz iff the function $H$ is independent of the direction $y$ in the first $S O(4)$ subset and transverse to the D5-brane. Hence, we see that the restricted form of ansatz forces us to smear the D-brane configuration along this direction. Finally, the last embedding does not impose any restriction on $H$. In the absence of CS terms, the RR Bianchi identities imply that 
the (smeared) function $H$ is harmonic in the space transverse to D-brane. This is compatible with the dilaton equation.

Finally, let us analyze the Einstein equation. As already pointed out, the pp-wave function $S$ appears only in the uu component of the Ricci tensor; all other components are the same as for a D-brane in a flat background. Note that the RR flux of the pp-wave contributes only to the $T_{u u}^{R R}$ component of the energy momentum tensor. This can easily be seen by realising that the only component of the inverse metric which involves the index $u$ is $g^{u v}$; hence, since $F_{[5]}$ does not contain the index $v$, there is no way in which one can contract the index $u$. Therefore, to be consistent with our ansatz, one must check that there are no extra source terms in other components of the energy momentum tensor coming from the cross-terms between the RR 5 -form of the wave and the RR fields of the D-brane. This is obviously true for any D-brane except the D3-brane. In this case, the possible embeddings are $(+,-, 2,0)$ and $(+,-, 1,1)$. In the second configuration, all cross-terms vanish identically. However, in the first case, there are non-vanishing cross-terms

$$
T_{u y^{1}} \sim \partial_{y^{2}} H, \quad T_{u y^{2}} \sim \partial_{y^{1}} H .
$$

Hence we see that for an $(+,-, 2,0)$ embedding, in order to satisfy the equations of motion, we are again forced to smear the function $H$ in the directions $y^{1}$ and $y^{2}$.

Provided that one imposes this constraint, the only nontrivial component of the Einstein equation is the $u u$ component

$$
-\frac{1}{2} S H^{-2}\left(\partial_{\vec{y}}^{2}+\partial_{\vec{y}^{\prime}}^{2}\right) H+H^{-1}\left(\partial_{\vec{y}}^{2}+\partial_{\vec{y}^{\prime}}^{2}\right) S+\left(\partial_{\vec{x}}^{2}+\partial_{\vec{x}^{\prime}}^{2}\right) S=\frac{1}{2} W^{2} H^{\frac{m-n-2}{2}}
$$

while all the other equations of motion and Bianchi identities reduce to

$$
\left(\partial_{\vec{y}}^{2}+\partial_{\vec{y}^{\prime}}^{2}\right) H=0
$$

\section{Supersymmetry analysis}

We now want to show that our ansatz preserves the same amount of supersymmetry as predicted by the probe analysis [29]. To make the parallel between the supersymmetry analysis in the supergravity and the probe brane approaches clear, we give some of the probe brane result in appendix B. The smeared solutions in Brinkman coordinates describe an array of flat $(+,-, m+2, m)$ branes. Therefore, from the D-brane probe point-of-view [29], we expect them to preserve only one quarter of the supersymmetries. The $(+,-, m, m)$ branes should lead to non-supersymmetric

solutions, except for the $(+,-, 0,0)$ D-string which should preserve one quarter of the supersymmetries.

Specialising the supersymmetry variations (2.7) to our ansatz gives the following set of equations. First, the dilatino variation reads

$$
\delta \chi=\left(\partial_{a} H \gamma^{\underline{a}}+\partial_{A} H \gamma^{\underline{A}}\right)\left(1-\gamma^{\underline{u v x^{1} \cdots x^{m} x^{1} \cdots x^{\prime n}}} \mathcal{P}_{k}\right) \epsilon=0,
$$


where $p=2 k+1$. For $k$ even, $\mathcal{P}_{k}=\sigma^{1}$ and for $k$ odd, $\mathcal{P}_{k}=i \sigma^{2}$. Underlined indices correspond to tangent space indices. So we see that one has to impose the standard projection condition for D-branes in flat space

$$
\gamma^{\underline{u v x x^{\prime}}} \mathcal{P}_{k} \epsilon=\epsilon .
$$

Here we have introduced the short notation

$$
\gamma^{\underline{u v x x^{\prime}}} \equiv \gamma^{\underline{u v x^{1} \cdots x^{m} x^{1} \ldots x^{\prime n}}},
$$

and similarly for the other combinations of $x, x^{\prime}, y$ and $y^{\prime}$.

Using the identity $\left(\gamma^{\underline{u}}\right)^{2}=0$, one shows that the gravitino variation in the direction $v$ simply reduces to the condition

$$
\partial_{v} \epsilon=0 .
$$

The gravitino variations in the directions $\hat{a}=(a, A)$ read

$$
\delta \Psi_{\hat{a}}=\partial_{\hat{a}} \epsilon+\frac{1}{8} H^{-1}\left(\partial_{\hat{a}} H\right) \epsilon+\frac{1}{8} W H^{\frac{m-n}{4}} \gamma^{\underline{u x y}} \gamma_{\underline{\hat{a}}}\left(i \sigma_{2}\right) \epsilon=0 .
$$

Instead of trying to solve this equation directly, it is instructive to first consider the integrability condition coming from the gravitino variation in these directions

$$
\left[\delta \Psi_{\hat{a}}, \delta \Psi_{\hat{b}}\right]=\partial_{[\hat{a}}\left(W H^{\frac{(m-n)}{4}}\right) \gamma_{\hat{b}]} \gamma^{\underline{u}}\left(i \sigma_{2}\right) \epsilon=0 .
$$

We first try to satisfy this condition by imposing that

$$
W H^{\frac{(m-n)}{4}}=\text { const. }
$$

We will now discuss separately the cases $m \neq n$ and $m=n$.

$\underline{m \neq n}:$

In this case, using (2.14), the condition (3.7) implies that $W(y)^{1 / 2} \hat{H}\left(y^{\prime}\right)^{1 / 2}$ is a constant. This is true only if $W$ and $\hat{H}$ are constant and therefore, if $H$ is trivial. In order to satisfy (3.6) with a nontrivial $H$, we are forced to impose

$$
\gamma^{\underline{u}} \epsilon=0 .
$$

We still have to check whether satisfying the integrability condition is enough to ensure that the supersymmetry variation of $\Psi_{\hat{a}}$,

$$
\delta \Psi_{\hat{a}}=\partial_{\hat{a}} \epsilon+\frac{1}{8} H^{-1}\left(\partial_{\hat{a}} H\right) \epsilon=0,
$$

is satisfied. The solution to this equation is

$$
\epsilon=H^{-\frac{1}{8}} \hat{\epsilon}\left(u, x, x^{\prime}\right) .
$$


Using the condition (3.8), the gravitino variation in the $\hat{\imath}=(i, I)$ directions becomes trivial $\partial_{\hat{\imath}} \epsilon=0$, while the variation in the $u$ direction reduces to

$$
\delta \Psi_{u}=\partial_{u} \epsilon(u)+\frac{1}{8} W H^{\frac{(m-n-2)}{4}} \gamma \frac{u x y}{\underline{\underline{v}}} \mathcal{P}_{k} \hat{\epsilon}(u)=0
$$

Since $\hat{\epsilon}$ does not depend in $y, y^{\prime}$, this equation has a nontrivial solution only if

$$
W H^{\frac{(m-n-2)}{4}}=W(y)^{\frac{m-n+2}{2(m-n)}} \hat{H}\left(y^{\prime}\right)^{\frac{m-n-2}{2(m-n)}}=\text { const. },
$$

which further implies

$$
\begin{aligned}
& m-n=2 \quad W(y)=\text { const. } \quad \text { or } \\
& n-m=2 \quad \hat{H}(y)=\text { const. }
\end{aligned}
$$

In other words, we see that the $(+,-, m, m \pm 2)$ embeddings will be supersymmetric only if we smear the function $H$ in part of the transverse space. These 1/4-BPS configurations are summarised in table (11).

$\underline{m=n}:$

In this case, the condition (3.7) implies that $W$ is constant, which is consistent with the condition (2.15). The gravitino variation $\delta \Psi_{\hat{a}}$, reduces to

$$
\delta \Psi_{\hat{a}}=\partial_{\hat{a}} \epsilon+\frac{1}{8} H^{-1}\left(\partial_{\hat{a}} H\right) \epsilon+\frac{1}{8} W \gamma \frac{u x y}{\underline{\hat{a}}}\left(i \sigma_{2}\right) \epsilon=0
$$

which can now be integrated, yielding the spinor

$$
\epsilon=H^{-\frac{1}{8}}\left(1-\frac{1}{8} W \gamma \frac{u x y}{a} y^{a} \gamma_{\underline{a}}\left(i \sigma_{2}\right)\right) \hat{\epsilon}\left(u, x, x^{\prime}\right)
$$

Using this expression in the gravitino variations in the directions $\hat{\imath}=(i, I)$ give

$$
\partial_{\hat{\imath}} \hat{\epsilon}\left(u, x, x^{\prime}\right)+\frac{1}{8} W H^{-\frac{1}{2}} \gamma \frac{u x y}{\underline{\hat{\imath}}} \gamma_{\underline{\hat{n}}}\left(i \sigma_{2}\right) \hat{\epsilon}\left(u, x, x^{\prime}\right)=0 .
$$

Since $\hat{\epsilon}$ does not depend on $y, y^{\prime}$ the only way this equation can be satisfied is if we again impose the condition (3.8). Here we recover the same constraint as in the D-brane probe analysis described in the appendix, where equation (B.19) is also consequence of the existence of worldvolume coordinates $x^{\hat{\imath}}$.

Finally, the supersymmetry variation in the direction $u$ reduces to

$$
\partial_{u} \epsilon(u)+\frac{1}{4} W H^{-\frac{1}{2}} \gamma \frac{x y}{2}\left(i \sigma_{2}\right) \epsilon(u)=0,
$$

which obviously does not have any solutions. Hence the $(+,-, m, m)$ embeddings of the D-branes break all supersymmetries! This is precisely the result predicted by the D-brane probe analysis and we see that supersymmetry fails for the same reason in 
both approaches. Namely, in both cases it is not possible to simultaneously satisfy the constraints coming from the supersymmetry/kappa symmetry conditions in the $u$ and $x^{\hat{\imath}}$ directions.

The previous discussion does not hold if there are no worldvolume directions $x^{\hat{\imath}}$, namely for the D-string. In this case, equation (3.16) is missing and therefore one does not need to impose the projection (3.8) anymore. The variation in the $u$ direction now becomes

$$
\partial_{u} \hat{\epsilon}(u)-\frac{1}{2} H^{-\frac{1}{2}}\left[\left(\partial_{\hat{a}} S\right)-\frac{1}{16} W^{2} y^{a}\right] \gamma^{\frac{u \underline{\hat{a}}}{\hat{\epsilon}}}+\frac{1}{8} W H^{-\frac{1}{2}} \gamma^{\underline{y}}\left[\left(\gamma^{\underline{u v}}+\eta^{\underline{u v}}\right) i \sigma_{2}\right] \hat{\epsilon}=0 .
$$

The content of the first bracket gives the BPS equation for $S$,

$$
\partial_{\hat{a}} S-\frac{1}{16} W^{2} y^{\hat{a}}=0,
$$

while the content of second bracket, when combined with (3.2) implies the condition

$$
\left(1+\sigma_{1}\right) \hat{\epsilon}=0 .
$$

In conclusion, the D-string preserves exactly one quarter of the supersymmetries.

\begin{tabular}{|c|c|c|}
\hline brane & embedding & susy \\
\hline D1 & $(+,-, 0,0)$ & $1 / 4$ \\
\hline \multirow[t]{2}{*}{ D3 } & $(+,-, 2,0)$ & $1 / 4$ \\
\hline & $(+,-, 1,1)$ & - \\
\hline \multirow[t]{2}{*}{ D5 } & $(+,-, 3,1)$ & $1 / 4$ \\
\hline & $(+,-, 2,2)$ & - \\
\hline \multirow[t]{2}{*}{ D7 } & $(+,-, 4,2)$ & $1 / 4$ \\
\hline & $(+,-, 3,3)$ & - \\
\hline
\end{tabular}
Again, let us emphasise that the D-brane probe discussion is completely parallel. All the results of this supersymmetry analysis are summarised in table 1 .

Table 1: Amount of supersymmetry preserved by the various D-brane supergravity solutions.

\section{Solving the field equations}

In this section we solve the equations of motion (2.19) and (2.20). For all solutions (except the D-string), the presence of the D-brane modifies the function $S$ which 
characterises the pp-wave. On the other hand, for our ansatz, the field equations imply that the function $H$ (which specifies the D-brane) is completely unmodified by the presence of the pp-wave. Therefore, this ansatz does not catch the back-reaction of the pp-wave on the D-brane. For a generic embedding of the D-brane, one expects that the (fully localised) D-brane is modified by the pp-wave. However, as our fully localised, nonsupersymmetric solutions demonstrate, this does not have to hold for some specific embeddings.

We will divide the discussion of the solutions according to the number of supersymmetries that they preserve, describing first the 1/4-BPS D-branes; we can split them into two subclasses : unsmeared solutions (D1 and $D 7)$ and smeared ones (D3 and $D 5$ ). Then, we turn to the integration of equations (2.20) and (2.19) for the non-supersymmetric $(+,-, m, m)$-embeddings.

\subsection{Supersymmetric solutions}

\section{D-string :}

The D-string has Neumann boundary conditions along the lightcone coordinates $u$ and $v$ and Dirichlet boundary conditions along the space transverse to the pp-wave. In this case, equations (2.19) and (2.20) are fully decoupled and hence the pp-wave and the D-string are completely insensitive to the presence of each other. Explicitly, the solution is given by

$$
\begin{aligned}
& H=1+\frac{Q}{\left(y^{2}+y^{\prime 2}\right)^{3}} \\
& S=\frac{1}{32} W^{2}\left(y^{2}+y^{\prime 2}\right), \quad W=\text { const. } .
\end{aligned}
$$

Smeared D-branes:

The supersymmetry analysis performed above leads to the conclusion that $D 3$ and $D 5$-branes with the respective embeddings $(+,-, 2,0)$ and $(+,-, 3,1)$ are supersymmetric only if the function $H$ does not depend on the coordinates $y$ transverse to the brane that belong to the first $S O(4)$ subspace. The harmonic functions $H$ in this case are

$$
D 3: \quad H=1+\frac{Q}{\left|y^{\prime}\right|^{2}}, \quad \text { D5 }: \quad H=1+\frac{Q}{\left|y^{\prime}\right|} .
$$

We are interested in solutions which asymptotically, far away from the D-brane, reproduce the Hpp-wave vacuum (2.1). To get a solution with this property, one can choose to decompose $S$ as the sum of a function which depends on $x$ and a function which depends on $y$

$$
S\left(x, x^{\prime}, y, y^{\prime}\right)=S_{x}(|x|)+S_{x^{\prime}}\left(\left|x^{\prime}\right|\right)+S_{y}(|y|)+S_{y^{\prime}}\left(\left|y^{\prime}\right|\right)
$$


With this ansatz the $x$-dependent and $x^{\prime}$-dependent parts of the equation (2.19) must vanish separately. Imposing that the solution reduces asymptotically to (2.1) fixes the functions $S_{x}$ and $S_{x^{\prime}}$,

$$
S_{x}+S_{x^{\prime}}=\frac{1}{32} W^{2}\left(|x|^{2}+\left|x^{\prime}\right|^{2}\right)
$$

up to a homogeneous solution of the Laplace equation. This part would correspond to a pure gravitational pp-wave propagating along the worldvolume of the D-brane (and smeared in the transverse directions). Since we are not interested in pure gravitational pp-waves, we have chosen to set this part to zero in (4.4). For the same reasons we will, in what follows, always ignore this kind of freedom when solving the equation for $S$.

To write an ansatz for the $y, y^{\prime}$-dependent parts of the metric, we will assume that the pp-wave is modified only in the directions transverse to the brane and the directions of smearing. ${ }^{6}$ Together with the asymptotic conditions, this assumption leads to the following formula

$$
S_{y}+S_{y^{\prime}}=\frac{1}{32} W^{2}\left(|y|^{2}+\left|y^{\prime}\right|^{2}\right)+f\left(\left|y^{\prime}\right|^{2}\right) .
$$

where $f$ is determined by plugging (4.4) and (4.5) into the field equation (2.19). The solutions are given by

$$
\begin{aligned}
D 3: & f=\frac{3}{16} Q W^{2} \ln \left|y^{\prime}\right|, \\
D 5: & f=\frac{1}{8} Q W^{2}\left|y^{\prime}\right| .
\end{aligned}
$$

Let us now examine some of their properties. First, since $\ln \left|y^{\prime}\right|,\left|y^{\prime}\right|$ grow slower than the quadratic terms in the pp-wave $S$ function, it is clear that both solutions asymptotically reduce to (2.1), as required. So although it looks like the deformation of the pp-wave due to the D-brane grows (and blows up) at infinity, this is just an artifact of the coordinates in which the pp-wave is written.

Second, it seems that in the case of the $D 3$-brane, the corrections to the pp-wave $S$ function blow up as we approach the D-brane. To explore whether this is indeed true, we look for possible invariant quantities that one can construct from the metric. The Ricci scalar is finite everywhere and independent of $S$. Other quantities (like $\left.R_{\mu \nu \xi \eta} R^{\mu \nu \xi \eta}, R_{\mu \nu} R^{\mu \nu}\right)$ are also independent of $S$, and diverge for small $r$. The only quantity which depends on $S$ is given by $R_{\mu \nu} \xi^{\mu} \xi^{\nu}$, where $\xi=\partial_{u}$ is the timelike Killing vector field. By looking at the $R_{u u}$ component in the appendix, one can easily see

\footnotetext{
${ }^{6}$ Since the direction along which we smear is not a direction of isometry of the wave (and since gravity is a highly nonlinear theory), it is not a priori clear that this assumption should hold. However, we will see that it leads to a physically acceptable solution.
} 
that this scalar also diverges for small $r$. Note that the divergent term in this scalar originates from corrections to the $S$ functions.

We now want to see whether these quantities diverge along timelike/null geodesics in finite proper time. So let us consider the geodesics for a radially infalling particle. To write down the equation of the geodesics, we use of the fact that $\xi=\partial_{u}$ and $\eta=\partial_{v}$ are Killing vectors with corresponding conserved quantities : the light-cone energy $p_{+}$and the light-cone momentum $p_{-}$,

$$
(\xi)_{\mu} p^{\mu}=H^{-\frac{1}{2}}(\dot{v}+2 S \dot{u})=p_{+}, \quad(\eta)_{\mu} p^{\mu}=H^{-\frac{1}{2}} \dot{u}=p_{-} .
$$

Here dots denote derivatives of the coordinates with respect to the proper time. ${ }^{7}$ Using these quantities, one can eliminate $\dot{u}$ and $\dot{v}$ from the action of the point particle. Furthermore, one can check that it is consistent with the equations of motion to set $x=x^{\prime}=y=0$. Plugging this back into the metric we get

$$
\epsilon=p_{+} p_{-} H^{\frac{1}{2}}-2 p_{+}^{2} H^{\frac{1}{2}} S-H^{\frac{1}{2}} \dot{y}^{2},
$$

where $\epsilon=-1,0,1$ for spacelike, null and timelike geodesics respectively. Hence the equations of motion for the radially infalling particle reduce to

$$
\left(\dot{y}^{\prime}\right)^{2}=\frac{-\epsilon}{\sqrt{1+\frac{Q}{\left(y^{\prime}\right)^{2}}}}+2 p_{-}\left(p_{+}-\frac{1}{32} W^{2} p_{-} y^{\prime 2}-\frac{3}{16} Q W^{2} p_{-} \ln \left|y^{\prime}\right|\right) .
$$

We see that by setting $Q=0$ one recovers the equation for the linear harmonic oscillator, as expected. If we have $W=0$, the previous equation can be integrated for small $y^{\prime}$, leading to conclusion that a radially infalling particle can reach the singularity in finite proper time. A direct inspection of (4.9) for small $y^{\prime}$ reveals that turning on $W$ makes the proper time in which the particle reaches the singularity shorter. In conclusion, the presence of the pp-wave strengthens the attraction of the singular smeared D3-brane solution.

D7-brane:

Finally, we can also write down a supergravity solution which describes a D7brane with the $(+,-, 4,2)$ embedding. In this case, the smearing of the harmonic function $H$ is not required. Hence, one gets a fully localised solution

$$
\begin{aligned}
H & =1+Q \ln \left|y^{\prime}\right| \\
S & =\frac{1}{32} W^{2}\left(x^{2}+x^{\prime 2}+y^{2}+y^{\prime 2}\right)-\frac{2}{32} W^{2} Q\left|y^{\prime}\right|^{2}\left(\ln \left|y^{\prime}\right|-1\right) .
\end{aligned}
$$

The unexpected and puzzling feature of this solution is that despite being fully localised, it still breaks more supersymmetries than the half predicted by the probe

\footnotetext{
${ }^{7}$ We are choosing the parameter along the geodesic to be equal to the proper time.
} 
analysis [29] or the CFT approach [30]. There are several subtle points that might occur when one looks at D7-brane supergravity solutions, and which were not taken into account when constructing this solution. We did not attempt to fully resolve this apparent mismatch. Instead, we will comment on possible resolutions in the last section.

\subsection{Non-BPS solutions}

In this section we construct the non-supersymmetric solutions of the equations (2.19) and $(2.20)$. The classification made in section 3 implies that the D-branes described by our ansatz, with $(+,-, m, m)$ embeddings (for $m \neq 0$ ) are non-supersymmetric. The supergravity analysis agrees with the result obtained in the appendix, using D-brane probes.

Because of the very simple ansatz that we have, the equations of motion can be integrated directly, yielding the following solutions

- D3 $(+,-, 1,1)$ :

$$
\left\{\begin{array}{l}
H=1+\frac{Q}{\left(y^{2}+y^{\prime 2}\right)^{2}} \\
S=\frac{1}{32} W^{2}\left(x^{2}+x^{\prime 2}+y^{2}+y^{\prime 2}+\frac{Q}{\left(y^{2}+y^{\prime 2}\right)}\right)
\end{array}\right.
$$

- D5 $(+,-, 2,2)$ :

$$
\left\{\begin{array}{l}
H=1+\frac{Q}{y^{2}+y^{\prime 2}} \\
S=\frac{1}{32} W^{2}\left(x^{2}+x^{\prime 2}+y^{2}+y^{\prime 2}\right)-\frac{W^{2} Q}{8} \ln \left(y^{2}+y^{\prime 2}\right)
\end{array}\right.
$$

- $\mathrm{D} 7(+,-, 3,3)$ :

$$
\left\{\begin{array}{l}
H=1+\frac{Q}{2} \ln \left(y^{2}+y^{\prime 2}\right) \\
S=\frac{1}{32} W^{2}\left(x^{2}+x^{\prime 2}+y^{2}+y^{\prime 2}\right)-\frac{2 W^{2} Q}{32}\left(y^{2}+y^{\prime 2}\right)\left(\frac{1}{2} \ln \left(y^{2}+y^{\prime 2}\right)-1\right)
\end{array}\right.
$$

Let us now analyse the properties of these solutions. First we see that, contrary to the supersymmetric D-branes, all solutions are fully localised. Second, as expected, the D3 and D5-brane solutions asymptotically reduce to the Hpp-wave (2.1). The only case for which this is not true is the D7-brane, which again exhibits special features. Third, the correction terms to the function $S$ again blow up for small $y$, $y^{\prime}$. To see whether this leads to physical singularities, let us perform an analysis similar to the one we did in the supersymmetric cases but now on the example of the non-supersymmetric D3-brane. In this case all scalar quantities, including $R_{\mu \nu} \xi^{\mu} \xi^{\nu}$ 
(which is again the only quantity dependent on $S$ ) are finite. The equations of motion for a radially infalling particle reduce to

$$
\dot{r}^{2}=\frac{-\epsilon}{\sqrt{1+\frac{Q}{r^{4}}}}+2 p_{+} p_{-}-\frac{1}{16} W^{2} p_{-}^{2} r^{2}\left(1+\frac{Q}{r^{4}}\right)
$$

where $r^{2}=y^{2}+y^{\prime 2}$. If we compare this expression to the radially infalling particle (4.9) we see that the correction to the function $S$ (the term proportional to $\left.W^{2} Q\right)$ now has the opposite sign. This term becomes dominant for small $r$, causing a repulsive behaviour. While the pure pp-wave causes the focusing of the geodesics towards the $r=0$ geodesics, and the pure $D 3$-brane also acts as an attractor towards the $r=0$ point, the superposed system exhibits a repulsive behaviour! Note that this is opposite to the situation we had for the supersymmetric D3-brane, where the pp-wave was strengthening the attractive behavior of the source.

Finally, since these D-branes are non-supersymmetric, one should also raise the issue of the stability. Since these D-branes carry a conserved charge they for sure cannot decay into the vacuum. Moreover, a non-supersymmetric D-brane with the same orientation probing this background does not feel any force. These two properties could point towards the stability of these embeddings but, clearly, this problem deserves a separate study and investigation is currently in progress. In the next section we will propose several possible ways to address this issue in detail.

\section{Discussion}

To conclude, let us discuss some open questions and problems which certainly deserve further study.

The first set of problems concerns other supergravity solutions for D-branes in ppwaves. The supersymmetric D3 and D5-branes solutions that have been constructed in this paper are smeared. As for the cases of D-brane intersections in Minkowski space, this restriction is obviously a consequence of the oversimplified form of the ansatz which has been used. However, we believe that Brinkman coordinates and our ansatz should serve as a good starting point for the construction of more complex ansatzes for the fully localised D-brane solutions.

It would be also interesting to find supergravity solutions corresponding to the families of $1 / 2$ and $1 / 4$-BPS D-branes with nonvanishing worldvolume fluxes that were identified in [29]. This problem is presumably more complex than the previous

one, since the presence of worldvolume fluxes would lead to additional nontrivial bulk fluxes at the level of the supergravity solution.

An apparent puzzle that appeared in this work concerns the fully localised, supersymmetric D7-brane solution (4.13). Only in this case does the number of supersymmetries predicted by the probe brane/CFT analysis not match with the number 
of supersymmetries preserved by our solution. One speculative resolution of this paradox is the following. Since the probe approach does not take into account the back-reaction of the D-brane on the pp-wave background, some (super)symmetries might be absent when this effect is incorporated. In particular we suspect that ("supernumerary") supersymmetries of the supergravity solution which in the dual gauge theory are related to the superconformal symmetries will be absent due to the back-reaction of the D7-brane on the pp-wave. If this is indeed true then a similar type of mismatch should already appear between the supergravity solution for the D7-brane wrapping the $A d S_{5} \times S^{5}$ space and the D-brane probe analysis in the same background. The probe analysis of [29] for this case implies that the number of supersymmetries is sixteen. The supergravity solution is unfortunately not fully known [37, 38]. However, the expected dual field theory is the $\mathcal{N}=2$, $S U(N)$ gauge theory with $M$ hypermultiplets in the fundamental representation and one hypermultiplet in the adjoint representation of the gauge group. If this theory, whose one-loop beta function is proportional to the number of $D 7$-branes, breaks all superconformal supersymmetries at the quantum level, then the corresponding supergravity dual does not possess any supernumerary supersymmetries and preserves only eight supercharges.

To close the discussion of the D7-brane puzzle with a less speculative comment, let us mention that (super)conformality in the presence of D7-branes can be restored by canceling their induced RR-charge. A way to do this is to add orientifold 7-planes. One O7-plane cancels the charge of 4 D7-branes. In particular, if these charges are locally canceled, the dilaton is constant and the only effect of the orientifold is to make a $\mathbb{Z}_{2}$ identification on the transverse space of the D7-branes. Therefore, the supergravity solution for this system in a Hpp-wave is an unmodified Hpp-wave solution, up to the orbifold identification. Due to this identification, half of the supersymmetries are projected out, and hence the supergravity solution preserves one half of the supersymmetries, which is the same as the (O7/4 D7)-wave-system.

Another set of questions concerns the class of the non-supersymmetric solutions. First, all these solutions are fully localised. It is not clear to us why is this the case. Naively, one would expect (from the experience one has in flat space) that the simplest solutions should be those which are characterised by the highest number of (super)symmetries. Here, we seems to have an interesting counterexample of this "intuition". This, of course, might be a mere consequence of the very specific coordinates that we were using; there could be another set of coordinates in which fully localised 1/4-BPS solutions would look very simple. However, we still believe that it is an observation one should keep in mind when addressing similar problems of D-branes in other nontrivial spaces.

Second, these non-supersymmetric solutions, as many other solutions constructed in the literature, display a repulsion type of behaviour. Here, however for the D3brane solution, contrary to standard cases [39, 40], there is no curvature singularity 
present behind the repulsion radius. Except for this strange behavior it does not seem to exhibit any other unphysical behaviour.

Third, since the presented solution is non-supersymmetric, it could be unstable. Notice that the breaking of supersymmetries is due to the "bad" orientation of the D-brane in the RR flux of the pp-wave. One can speculate that possible tachyonic modes would lead to the change of the orientation of the D-brane in the pp-wave background, and that the final configuration would be a D-brane with a "good" orientation, which preserves some fraction of supersymmetry. To put this on a firmer ground, one would have to study these non-supersymmetric D-branes in more detail, using for instance the CFT or probe effective action points-of-view to see if their spectra contain tachyonic open string modes. If there are no tachyonic modes present and if these non-BPS D-branes are stable, then they could have an interesting new role in the context of nonsupersymmetric defect AdS/CFT correspondence. We are currently investigating this direction.

Finally, let us close this article by saying that we were studying, from the supergravity point of view, examples of D-brane embeddings which have been described in the literature using other approaches [29, 30]. However, it is very likely that there are many other (non)BPS D-brane embeddings in the Hpp-wave background. A systematic classification of all these configurations is desirable.

\section{Acknowledgements}

We have benefited from discussions with C. Bachas, M. Blau, S. Kovacs, C. Nuñez, T. Ortín, G. Papadopoulos, K. Skenderis, N. Suryanarayana, M. Taylor, P. Townsend and T. Wiseman. We are specially grateful to K. Peeters for many valuble comments and suggestions. P.B. acknowledges PPARC for financial support. This work is also partially supported by EU contracts HPRN-CT-2000-00122 and HPRN-CT-200000148. M.Z. would like to thank CMS for hospitality during the final stage of this work. 


\section{A. Some technical details}

\section{Some useful supergravity equations and notations}

The dilaton equation is

$$
R+4(\partial \phi)^{2}-4 \nabla^{2} \phi+\frac{1}{2 \cdot 3 !} H^{2}=0
$$

The Einstein equations are (here the Ricci scalar has been eliminated with the use of the dilaton equation)

$$
R_{\mu \nu}=2 \nabla_{\mu} \nabla_{\nu} \phi-\frac{1}{4} H_{\mu \xi \rho} H_{n u}^{\xi \rho}+\frac{1}{4} e^{2 \phi} \sum_{n} \frac{(-1)^{n}}{(n-1) !} T_{\mu \nu}^{(n)}
$$

where $T^{[n]}{ }_{\mu \nu}$ are the energy-momentum tensors for the RR fields

$$
T_{[n] \mu \nu}=F_{[n] \mu} \xi_{1} \cdots \xi_{(n-1)} F_{[n] \nu \xi_{1} \cdots \xi_{(n-1)}}-\frac{1}{2 n} g_{\mu \nu} F_{[n]}^{2}
$$

where $T^{[n]}{ }_{\mu \nu}$ are the energy-momentum tensors for the RR fields

$$
T_{[n] \mu \nu}=F_{[n] \mu} \xi_{1} \cdots \xi_{(n-1)} F_{[n] \nu \xi_{1} \cdots \xi_{(n-1)}}-\frac{1}{2 n} g_{\mu \nu} F_{[n]}^{2}
$$

The Bianchi identities and the equations of motion for the RR fields can be written in compact form as 41]

$$
\left\{\begin{array}{r}
d F-H_{[3]} \wedge F=0, \\
d H_{[3]}=0, \\
d H_{[7]}+\frac{1}{2}^{\star} F \wedge F=0
\end{array}\right.
$$

and

$$
\left\{\begin{array}{r}
d^{\star} F+H_{[3]} \wedge F=0, \\
d\left(e^{-2 \phi \star} H_{[3]}\right)+\frac{1}{2}^{\star} F \wedge F=0, \\
d\left(e^{2 \phi \star} H_{[7]}\right)=0,
\end{array}\right.
$$


where we are using the notation of [42, 43] in which forms of different degrees are formally combined into a single entity:

$$
\left\{\begin{array}{l}
C=C_{[0]}+C_{[1]}+C_{[2]}+\ldots, \\
F=F_{[1]}+F_{[2]}+F_{[3]}+\ldots
\end{array}\right.
$$

where the dual potentials are defined by the relations between field strengths

$$
\left\{\begin{array}{c}
F_{[10-n]}=(-1)^{[n / 2] \star} F_{[n]}, \\
H_{[7]}=e^{-2 \phi \star} H_{[3]},
\end{array}\right.
$$

\section{Vierbein, spin connection and Ricci tensor}

We choose the following vielbeins $e^{\underline{m}}=e_{\mu} \underline{\underline{m}} d x^{\mu}$

$$
\begin{aligned}
& e_{\underline{v}}=e^{\underline{u}}=H^{-\frac{1}{4}} d u, \quad e_{\underline{u}}=e^{\underline{v}}=H^{-\frac{1}{4}}(d v+S d u), \\
& -e_{\underline{i}}=e^{\underline{i}}=H^{-\frac{1}{4}} d x^{i}, \quad-e_{\underline{I}}=e^{\underline{I}}=H^{-\frac{1}{4}} d x^{I}, \\
& -e_{\underline{a}}=e^{\underline{a}}=H^{\frac{1}{4}} d y^{a}, \quad-e_{\underline{A}}=e^{\underline{A}}=H^{\frac{1}{4}} d y^{A}
\end{aligned}
$$

and the inverse vielbeins $\theta_{\underline{m}}=e_{\underline{m}}^{\mu} \partial_{\mu}$

$$
\begin{array}{rlrl}
\theta_{\underline{u}} & =H^{\frac{1}{4}}\left(\partial_{u}-S \partial_{v}\right), \theta_{\underline{v}}=H^{\frac{1}{4}} \partial_{v}, \\
\theta_{\underline{i}} & =H^{\frac{1}{4}} \partial_{i}, & \theta_{\underline{I}} & =H^{\frac{1}{4}} \partial_{I}, \\
\theta_{\underline{a}} & =H^{-\frac{1}{4}} \partial_{a}, & \theta_{\underline{A}} & =H^{-\frac{1}{4}} \partial_{A}
\end{array}
$$

and we use flat light cone metric $\eta \underline{u v}=1, \eta \underline{i j}=-1$.

The corresponding spin connection is

$$
\begin{array}{ll}
\omega_{\underline{u u i}}=-H^{\frac{1}{4}} \partial_{i} S, & \omega_{\underline{u u I}}=-H^{\frac{1}{4}} \partial_{I} S, \\
\omega_{\underline{u u a}}=-H^{-\frac{1}{4}} \partial_{a} S & \omega_{\underline{u u A}}=-H^{-\frac{1}{4}} \partial_{A} S \\
\omega_{\underline{u v a}}=\frac{1}{4} H^{-\frac{5}{4}} \partial_{a} H, & \omega_{\underline{u v A}}=\frac{1}{4} H^{-\frac{5}{4}} \partial_{A} H \\
\underline{\omega_{i a j}}=H^{-\frac{5}{4}} \partial_{a} H \delta_{i j}, & \omega_{\underline{I a J}}=H^{-\frac{5}{4}} \partial_{a} H \delta_{I J}, \\
\omega_{\underline{i A j}}=H^{-\frac{5}{4}} \partial_{A} H \delta_{i j}, & \omega_{\underline{I A J}}=H^{-\frac{5}{4}} \partial_{A} H \delta_{I J}, \\
\omega_{\underline{\omega_{b c c}}}=\frac{1}{2} H^{-\frac{5}{4}} \eta_{a[b} \partial_{c]} H, & \omega_{\underline{a b C}}=\frac{1}{4} H^{-\frac{5}{4}} \eta_{a b} \partial_{C} H, \\
\omega_{\underline{A B c}}=\frac{1}{4} H^{-\frac{5}{4}} \eta_{A B} \partial_{c} H, \omega_{\underline{A B C}}=\frac{1}{2} H^{-\frac{5}{4}} \eta_{A[B} \partial_{C]} H .
\end{array}
$$


Finally, the components of the Ricci tensor for the metric (2.8) are given by

$$
\begin{aligned}
R_{v v} & =0, \\
R_{u u} & =\partial_{i} \partial^{i} S+H^{-1} \partial_{\hat{a}} \partial^{\hat{a}} S+\frac{(3-p)}{2} H^{-1} \partial_{\hat{a}} \partial^{\hat{a}} \log H, \\
& -\frac{1}{2} S\left(H^{-2} \partial_{a} \partial^{a} H-\frac{p-1}{2} H^{-1} \partial_{a} \log H \partial^{a} \log H\right), \\
R_{u v} & =-\frac{1}{4}\left(H^{-2} \partial_{a} \partial^{a} H-\frac{p-1}{2} H^{-1} \partial_{a} \log H \partial^{a} \log H\right), \\
R_{i j} & =-\frac{1}{4} \eta_{i j}\left(H^{-2} \partial_{a} \partial^{a} H-\frac{p-1}{2} H^{-1} \partial_{a} \log H \partial^{a} \log H\right), \\
R_{a b} & =\frac{1}{4} \eta_{a b} H^{-1} \partial_{c} \partial^{c} H-\frac{p-3}{2} H^{-1} \partial_{a} \partial_{b} H-\frac{p-1}{8} \eta_{a b} \partial_{c} \log H \partial^{c} \log H \\
& +\frac{3 p-7}{4} \partial_{a} \log H \partial_{b} \log H .
\end{aligned}
$$

\section{B. Probe analysis}

In the this appendix we repeat some of the D-brane probe analysis of $[29]^{8}$, in order to make contact with the supersymmetry analysis from the supergravity point of view as presented in the main text.

For negative chirality type IIB spinors,

$$
\Gamma^{(11)} \epsilon=-\epsilon
$$

the Killing spinors of the Hpp-wave are given by [3]

$$
\begin{aligned}
\epsilon(u, z)=\left(1-\frac{i}{2} \sum_{\mu=1}^{4} \gamma_{\underline{v}}\left(z^{\mu} \gamma_{\underline{\mu}} I+z^{\mu+4} \gamma_{\underline{\mu+4}} J\right)\right) \\
\left(\cos \frac{1}{2} u-i I \sin \frac{1}{2} u\right)\left(\cos \frac{1}{2} u-i J \sin \frac{1}{2} u\right) \chi
\end{aligned}
$$

where we have defined $I=\gamma_{\underline{1234}}, J=\gamma_{\underline{5678}}$. The matrices $\gamma_{\underline{u}}, \gamma_{\underline{v}}$ and $\gamma_{\underline{\mu}}(\mu=1, \ldots, 8)$ are tangent space, $32 \times 32$ Dirac matrices and the complex spinor $\chi$, subject to the chirality projection (B.1), can be decomposed as $\chi=\lambda+i \eta$, where $\lambda$ and $\eta$ are constant, real, negative chirality spinors.

In the absence of worldvolume fluxes, the kappa-symmetry projection operator, for a D-brane with the $(+,-, m, n)$ embedding, is given by

$$
\Gamma=\gamma_{\underline{u v a_{1} \ldots a_{m} b_{1} \ldots b_{n}}} K^{\frac{p+1}{2}} I \equiv Q K^{\frac{p+1}{2}} I,
$$

\footnotetext{
${ }^{8}$ The original discussion of [29] contained a mistake in counting the number of supersymmetries preserved by the D-branes with the embedding $(+,-, m, m)$ for $(m=1,2,3)$. This was corrected in the revised version. We are grateful to the authors of [29] for correspondence regarding this issue.
} 
where $K$ acts by complex conjugation and $I$ by multiplication by $-i$.

The amount of supersymmetry preserved by the D-brane probe can be determined from the condition

$$
\Gamma \epsilon=\epsilon,
$$

where $\epsilon$ is the Killing spinor of the background in the which probe is embedded, and it is evaluated on the worldvolume of the D-brane. For the D3-brane, the condition (B.4) reduces to

$$
\epsilon=-i Q \epsilon,
$$

while for the $D 1$ and $D 5$-branes, it reduces to

$$
\epsilon=i Q \epsilon^{*}
$$

Let use consider the D3-brane in detail for the two possible embeddings, $(+,-, 2,0)$ and $(+,-, 1,1)$. In the first case, the projection operator is $Q_{1}=\gamma_{\underline{u v 12}}$ while in the second case $Q_{2}=\gamma_{\underline{u v 15}}$. A crucial property to note is that $Q_{1}$ commutes with $I$ and $J$ while $Q_{2}$ anticommutes with $I$ and $J$.

Plugging the Killing spinor (B.2) into the equation (B.5) and imposing that the equation should hold for any $u$ lead to the following three $z$-dependent equations

$$
\begin{aligned}
& \left(1-\frac{i}{2} \sum_{a=1}^{4} \gamma_{\underline{v}}\left(z^{\mu} \gamma_{\underline{\mu}} I+z^{\mu+4} \gamma_{\underline{\mu+4}} J\right)\right) \chi= \\
& -i Q\left(1-\frac{i}{2} \sum_{a=1}^{4} \gamma_{\underline{v}}\left(z^{\mu} \gamma_{\underline{\mu}} I+z^{\mu+4} \gamma_{\underline{\mu+4}} J\right)\right) \chi \\
& \left(1-\frac{i}{2} \sum_{a=1}^{4} \gamma_{\underline{v}}\left(z^{\mu} \gamma_{\underline{\mu}} I+z^{\mu+4} \gamma_{\underline{\mu+4}} J\right)\right)(I+J) \chi= \\
& -i Q\left(1-\frac{i}{2} \sum_{a=1}^{4} \gamma_{\underline{v}}\left(z^{\mu} \gamma_{\underline{\mu}} I+z^{\mu+4}{\underline{\gamma_{\underline{\mu}+4}}} J\right)\right)(I+J) \chi \\
& \left(1-\frac{i}{2} \sum_{a=1}^{4} \gamma_{\underline{v}}\left(z^{\mu} \gamma_{\underline{\mu}} I+z^{\mu+4} \gamma_{\underline{\mu+4}} J\right)\right) I J \chi= \\
& -i Q\left(1-\frac{i}{2} \sum_{a=1}^{4} \gamma_{\underline{v}}\left(z^{\mu} \gamma_{\underline{\mu}} I+z^{\mu+4} \gamma_{\underline{\mu+4}} J\right)\right) I J \chi
\end{aligned}
$$

It is easy to see that, due to the identity $(I J)^{2}=1$, the first and the last equations are identical.

Next, we use the fact that the previous equations have to be satisfied for any value of the coordinates $x^{\hat{\imath}}$ along the D-brane worldvolume. Here, we will consider only D-branes sitting at the origin of the space in the Brinkman coordinates, and we choose the static gauge for the worldvolume directions. Therefore, all the coordinates 
transverse to the D-brane in $(\overline{B .7})$ can be set to zero and we only keep the dependence of the spinor on the worldvolume coordinates.

For the $(+,-, 2,0)$ embedding, we obtain the system of equations

$$
\begin{aligned}
& \chi=-i Q_{1} \chi \\
& (I+J) \chi=-i Q_{1}(I+J) \chi \\
& \gamma_{\underline{v}} \gamma_{\underline{a}} I \chi=-i Q_{1} \gamma_{\underline{v}} \gamma_{\underline{a}} I \chi \\
& \gamma_{\underline{v}} \gamma_{\underline{a}} I(I+J) \chi=-i Q_{1} \gamma_{\underline{v}} \gamma_{\underline{a}} I(I+J) \chi,
\end{aligned}
$$

where $a=1,2$ indices correspond to directions $x^{1}, x^{2}$ along which the D-brane extends. The last two equations come from the worldvolume dependent part of the equation (B.7).

Since for this embedding $I$ and $J$ commute with $Q_{1}$, equation $(\overline{\mathrm{B} .9})$ is by virtue of equation (B.8) automatically satisfied. Similarly, using (B.10), equation (B.11) is also satisfied. Finally, since $Q_{1}$ anticommutes with both $\gamma_{\underline{v}}$ and $\gamma_{\underline{i}}$ it is easy to see that if (B.8) holds, equation (B.10) is also satisfied. Hence, the only independent condition is obtained from the first equation (B.8). The real and imaginary parts of this equation both lead to the following constraint on the spinors

$$
\lambda=Q \eta .
$$

Hence, the $(+,-, 2,0)$ D3-brane sitting at the origin of the space breaks exactly one half of the supersymmetries of the Hpp-wave background.

Let us now consider the $(+,-, 1,1)$ embedding of the D3-brane. The kappasymmetry projection leads to the following set of equations

$$
\begin{aligned}
& \chi=-i Q_{2} \chi \\
& (I+J) \chi=-i Q_{2}(I+J) \chi \\
& \gamma_{\underline{v}} \gamma_{\underline{a}} I \chi=-i Q_{2} \gamma_{\underline{v}} \gamma_{\underline{a}} I \chi \\
& \gamma_{\underline{v}} \gamma_{\underline{a}} I(I+J) \chi=-i Q_{2} \gamma_{\underline{v}} \gamma_{\underline{a}} I(I+J) \chi,
\end{aligned}
$$

where now $a=1,5$. The last two sets of equations come from the worldvolume coordinates $\left(x^{1}=z^{1}, x^{1}=z^{5}\right)$ dependent part of the relations (B.7). First, since $I$ and $J$ now anticommute with $Q_{2}$, we see that the first and second equations are compatible only if we impose that

$$
(I+J) \chi=0 \text {. }
$$

This constraint implies that the equations (B.16) of the above system are trivially satisfied. Let us consider the third equation (B.15). Since $I, \gamma_{\underline{v}}$ and $\gamma_{\underline{a}}$ all anticommute with $Q_{2}$, after multiplication of the equation (B.15) with the (invertible) matrix $\gamma_{\underline{a}} I$, one obtains the condition

$$
\gamma_{\underline{v}} \chi=i \gamma_{\underline{v}} Q_{2} \chi
$$


which, when combined with (B.13), leads to

$$
\gamma_{\underline{v}} \chi=0 \text {. }
$$

However, this condition implies, together with the negative chirality condition for spinors (B.1) and the constraint (B.17), that $\chi=0$, as follows. By multiplying (B.17) with the invertible matrix $\gamma_{\underline{u v}} I$, after the use of $(\mathbb{B} .1)$, one gets the condition

$$
\gamma_{\underline{u v}} \chi=\chi
$$

which is compatible with $(B .19)$ only if $\chi=0$. Therefore, no supersymmetry is preserved by the D3-brane with embedding $(+,-, 1,1)$. A similar analysis for the $(+,-, 2,2)$ D5-brane or the $(+,-, 3,3)$ D7-branes leads to the same conclusions.

The D-string is different from higher dimensional $\mathrm{D} p$-branes since in this case there are no worldvolume directions transverse to the pp-wave. This means that the constraint $(\overline{B .19})$ associated to these worldvolume coordinates is absent, and one only has to impose the conditions $(\mathbb{B . 1 3})$ and (B.17). These two conditions can be satisfied simultaneously leading to the conclusion that the D-string is $1 / 4$-BPS.

\section{References}

[1] D. Berenstein, J. Maldacena and H. Nastase, Strings in flat space and pp-wave from $N=4$ super-Yang-Mills, hep-th/0202021.

[2] R. Güven, Plane wave limits and T-duality, Phys. Lett. B 482 (2000) 255, hep-th/0005061.

[3] M. Blau, J. Figueroa-O'Farrill, C. Hull and G. Papadopoulos, A new maximally supersymmetric background of IIB superstring theory, JHEP 0201 (2002) 047, hep-th/0110242.

[4] R. Penrose, Any spacetime has a plane wave as a limit, in Differential Geometry and relativity, pp.271-75, Reidel, Dordrecht, 1976.

[5] M. Cvetic, H. Lü and C.N. Pope, M-theory PP-Waves, Penrose Limits and Supernumerary Supersymmetries, hep-th/0203229.

[6] J. Gauntlett and C.M. Hull, pp-waves in 11-dimensions with extra supersymmetry, hep-th/0203255.

[7] J. Maldacena, The Large N Limit of Superconformal Field Theories and Supergravity, Adv. Theor. Math. Phys. 2 (1998) 231, hep-th/9711200.

[8] E. Witten, Anti-de Sitter space and holography, Adv. Theor. Math. Phys. 2, 253 (1998), hep-th/9802150. 
[9] S. S. Gubser, I. R. Klebanov and A. M. Polyakov, Gauge theory correlators from non-critical string theory, Phys. Lett. B 428, 105 (1998), hep-th/9802109.

[10] O. Aharony, S.S. Gubser, J. Maldacena, H. Ooguri and Y. Oz, Large $N$ field theories, string theory and gravity, Phys. Rep. 323 (2000) 183, hep-th/9905111.

[11] D.Berenstein and H.Nastase, On lightcone string field theory from super Yang-Mills and holography, hep-th/0205048

[12] R.R. Metsaev, Type IIB Green-Schwarz superstring in plane wave Ramond-Ramond background, Nucl. Phys. B 625 (2002) 70, hep-th/0112044.

[13] R.R. Metsaev and A.A. Tseytlin, Exactly solvable model of superstring in plave wave Ramond-Ramond backgrond, hep-th/0202109.

[14] M. Blau, J. Figueroa-O'Farrill, C. Hull and G. Papadopoulos, Penrose limits and maximal supersymmetry, hep-th/0201081.

[15] A. Fayyazuddin and D.J. Smith, Localised intersections of M5-branes and four-dimensional superconformal field theories, JHEP 9904 (1999) 030, hep-th/9902210.

[16] A. Fayyazuddin and D.J. Smith, Warped AdS near-horizon geometry of completely localised intersections of M5-branes, JHEP 0010 (2000) 023, hep-th/0006060.

[17] H.Lu and C.Pope, Interacting Intersections, Class. Quant. Grav. 15, 2239 (1998), hep-th/9710243.

[18] J. de Boer, A. Pasquinucci and K. Skenderis, AdS/CFT dualities involving large 2d $N=4$ superconformal symmetry, Adv. Theor. Math. Phys. 3 (1999) 577, hep-th/9904073.

[19] G. Papadopoulos, J. G. Russo and A. A. Tseytlin, Curved branes from string dualities, Class. Quant. Grav. 17, 1713 (2000), hep-th/9911253.

[20] D. Youm, Partially Localised Intersecting BPS Branes, hep-th/9902208.

[21] C. Bachas and M. Petropoulos, Anti-de-Sitter D-branes, JHEP 0102, 025 (2001), hep-th/0012234.

[22] J. M. Maldacena, G. W. Moore and N. Seiberg, Geometrical interpretation of D-branes in gauged WZW models, JHEP 0107, 046 (2001), hep-th/0105038.

[23] B. Ponsot, V. Schomerus and J. Teschner, Branes in the Euclidean AdS(3), JHEP 0202, 016 (2002), hep-th/0112198.

[24] M. Billó and I. Pesando, Boundary states for GS superstrings in an Hpp wave background, hep-th/0203028. 
[25] M. Alishahiha and M.M. Sheikh-Jabbari, Strings in PP-waves and Worldsheet Deconstruction, hep-th/0204174.

[26] A. Kumar, R.R. Nayak and Sanjay, D-Brane Solutions in pp-wave Background, hep-th/0204025.

[27] H. Singh, M5-branes with 3/8 supersymmetry in pp-wave background, hep-th/0205020.

[28] M. Cvetic, H. Lü and C.N. Pope, Penrose limits, pp-waves and deformed M2-branes, hep-th/0203082.

[29] K. Skenderis and M. Taylor, Branes in AdS and pp-wave spacetimes, hep-th/0204054.

[30] A. Dabholkar and S. Parvizi, Dp Branes in PP-wave Background, hep-th/0203231.

[31] C.S. Chu and P.M. Ho, Noncommutative D-brane and open string in pp-wave background with B-field, hep-th/0203186.

[32] D. Berenstein, E. Gava, J. Maldacena, K.S. Narain and H. Nastase, Open strings on plane waves and their Yang Mills duals, hep-th/0203249.

[33] P. Lee and J. Park, Open strings in pp-wave background from defect conformal field theory, hep-th/0203257.

[34] A. Gomberoff, D. Kastor, D. Marolf and J. Traschen, Fully Localised Brane Intersections - The Plot Thickens, Phys. Rev. D 61 (2000) 024012, hep-th/9905094.

[35] A. Tseytlin, Composite BPS configurations of p-branes in 10 and 11 dimensions, Class. Quant. Grav. 14 (1997) 2085, hep-th/9702163. [abs, src, ps, other] :

[36] E. Witten, Baryons And Branes In Anti de Sitter Space, JHEP 9807 (1998) 006, hep-th/9805112.

[37] A. Fayyazuddin and M. Spalinski, Large N Superconformal Gauge Theories and Supergravity Orientifolds, Nucl. Phys. B 535 (1998) 219, hep-th/9805096.

[38] O. Aharony, A. Fayyazuddin and J. Maldacena, The Large $N$ Limit of $\mathcal{N}=2,1$ Field Theories from Threebranes in F-theory, JHEP 9807 (1998) 013, hep-th/9806159.

[39] R. Kallosh and A. D. Linde, Exact supersymmetric massive and massless white holes, Phys. Rev. D 52, 7137 (1995), hep-th/9507022.

[40] C. V. Johnson, A. W. Peet and J. Polchinski, Gauge theory and the excision of repulson singularities, Phys. Rev. D 61, 086001 (2000), hep-th/9911161.

[41] B. Janssen, P.Meessen and T. Ortin, The D8-brane tied up:strings and brane solutions in massive type IIA supergravity, Phys. Lett. B453:229, 236 (1999), hep-th/9901078 
[42] M.Douglas, Branes within branes, hep-th/9512077

[43] M.Green, C.Hull and P.Townsend, D-brane Wess-Zumino actions, T duality and the cosmological constant Phys. Lett. B382 65, 1996, hep-th/9604119 